\title{
Gradhiva
}

GRADHIV

Revue d'anthropologie et d'histoire des arts

$31 \mid 2020$

L'idéal du musicien et l'âpreté du monde

\section{Au-delà du campement - une archéologie mémorielle de la frontière}

Yasmine Bouagga

\section{(2) OpenEdition}

1 Journals

Édition électronique

URL : http://journals.openedition.org/gradhiva/5181

DOI : 10.4000/gradhiva.5181

ISSN : 1760-849X

Éditeur

Musée du quai Branly Jacques Chirac

\section{Édition imprimée}

Date de publication : 2 septembre 2020

Pagination : 167-168

ISBN : 978-2-35744-131-6

ISSN : 0764-8928

Référence électronique

Yasmine Bouagga, "Au-delà du campement - une archéologie mémorielle de la frontière », Gradhiva

[En ligne], 31 | 2020, mis en ligne le 31 mars 2021, consulté le 02 avril 2021. URL : http://

journals.openedition.org/gradhiva/5181 ; DOI : https://doi.org/10.4000/gradhiva.5181

Ce document a été généré automatiquement le 2 avril 2021.

(c) musée du quai Branly 


\section{Au-delà du campement - une archéologie mémorielle de la frontière}

Yasmine Bouagga

\section{RÉFÉRENCE}

Dan Hicks et Sarah Mallet, Lande : The Calais 'Jungle' and Beyond, Bristol/Chicago, Bristol University Press, coll. «Bristol Shorts Research », 2019, 154 p. 


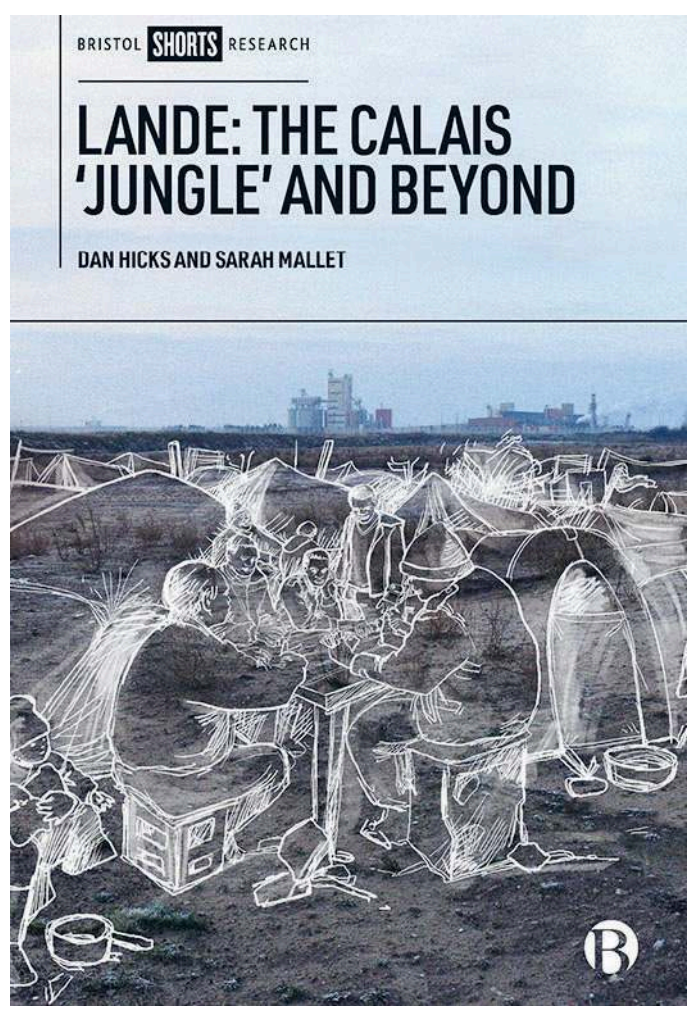

1 La «crise des réfugiés» de 2015-2016 aura suscité au moins autant d'attention médiatique que d'engagements artistiques et d'intérêts académiques. De nombreuses publications étudient le sort des migrants arrivant en Europe, et leurs conditions de vie dans les campements insalubres où ils sont bloqués à la frontière. La « jungle » de Calais (2015-2016) a été un terrain tout particulièrement investi par la recherche, l'investigation journalistique ou l'intervention artistique ${ }^{1}$. L'exposition suraiguë de ce lieu contraste avec sa brutale destruction, par bulldozers et incendies, en octobre 2016. Que reste-t-il de ce campement à la frontière ? C'est ce qu'ont cherché Dan Hicks et Sarah Mallet, à travers un programme d'archéologie du contemporain.

2 Initialement, il s'agissait de documenter les constructions et l'habitat des camps de réfugiés dans différents pays, de manière à comparer ces logements éphémères ${ }^{2}$. Mais au moment du démarrage du projet, les autorités françaises avaient commencé à détruire le campement. Il n'était plus possible d'étudier sa matérialité, les auteurs ont donc décidé d'étudier ses traces ${ }^{3}$. Ils s'inscrivent ici dans la filiation d'un courant de recherche fécond de la dernière décennie, qui a porté son attention sur les traces de la migration, pour en documenter les parcours et les épreuves : c'est le travail minutieux de Jason De León à la frontière entre les États-Unis et le Mexique ${ }^{4}$; ou encore celui de Charles Heller et de son équipe de Forensic Architecture pour retracer le parcours du «left-to-die-boat ", une embarcation de personnes migrantes abandonnées à la noyade en Méditerranée ${ }^{5}$. En utilisant les techniques de l'investigation criminelle, la recherche de témoignages, de preuves matérielles, de vestiges ou même de résidus, ces travaux révèlent la violence de l'expérience des frontières.

3 Dan Hicks et Sarah Mallet entendent aborder de la même façon le campement de la frontière franco-britannique, soulignant sa violence et son caractère létal. Chacun des cinq chapitres de l'ouvrage débute par la phrase "l'opération de démantèlement humanitaire est terminée ", prononcée par la préfète au moment de la destruction du 
bidonville. Le livre entreprend de déconstruire ce discours officiel, en mobilisant une réflexion théorique sur la frontière (borderline archeology), l'environnement hostile (environmental hostility), la violence temporelle (temporal violence); les deux derniers chapitres examinent les résistances à cette violence : une politique du visible (visual politics) et le don par l'engagement solidaire (giving time), qui opposent à la violence des assignations dans la précarité du campement des formes de permanence et même de monumentalité . Cette réflexion théorique envisage alors le lieu non pas comme camp mais comme paysage (landscape), au sens d'un territoire assemblant humains et nonhumains. Ce faisant, les auteurs soulignent l'inscription de ce campement éphémère dans une temporalité au long cours, celle des héritages coloniaux et des continuations d'empires, qui font de Calais une frontière britannique en France. En effet, Calais fut une ville anglaise au Moyen Âge, et les migrants qui tentent de traverser irrégulièrement sont issus d'anciens territoires de l'Empire colonial britannique (Soudan, Afghanistan, Irak, Somaliland...). C'est par la révélation de ces passés refoulés que les auteurs veulent, au-delà d'une anthropologie de l'infrastructure, exposer le sens « cosmopolitique » du campement.

Dans l'ouvrage, l'approche d'archéologie du contemporain signifie la recherche des continuités, des répétitions et des palimpsestes, par opposition à une démarche qui singulariserait le camp de réfugiés comme un événement. En effet, le lieu désigné officiellement comme le "campement de la Lande", et plus couramment comme la « jungle» (ou la " grande jungle») de Calais, ne fut que l'un des nombreux campements apparus à la frontière franco-britannique depuis la mise en place de contrôles côté français afin d'empêcher les migrations irrégulières vers la Grande-Bretagne. La multiplication de campements de familles réfugiées, principalement kosovares, à l'hiver 1999, avait conduit à l'ouverture à Sangatte d'un centre d'accueil humanitaire géré par la Croix-Rouge. Après sa fermeture, divers squats et campements se sont formés, comptant entre quelques dizaines et plusieurs centaines de personnes, apparaissant et disparaissant en fonction des cycles de migrations et d'expulsions. Le «campement de la Lande» ne fut que l'un des avatars de ces regroupements transitoires, mais certainement le plus spectaculaire par sa taille (jusqu'à dix mille personnes), par l'ampleur de l'engagement solidaire qui l'a doté d'une architecture et de traits matériels originaux (des cabanes en kit, un théâtre, des écoles...), et surtout par la très forte médiatisation dont il a fait l'objet. C'est donc un lieu particulier, néanmoins inscrit dans une longue histoire - une histoire dont les répétitions excèdent les migrations contemporaines, puisque le centre Jules-Ferry (centre de loisirs de la ville de Calais mis à disposition pour la distribution alimentaire aux personnes migrantes en transit) fut précisément le lieu d'accueil des réfugiés espagnols en 1939 ; il était alors situé à l'ouest de la ville, où les réfugiés, placés en quarantaine, avaient interdiction de se rendre ${ }^{7}$. Au regard de ces éléments, l'archéologie du contemporain pourrait être une démarche fructueuse pour révéler les différentes strates qui composent les agencements matériels, sociaux ou institutionnels. Lande: The Calais 'Jungle' and Beyond ne tient toutefois pas ses promesses, souffrant du manque de données empiriques, d'analyse des matérialités, d'attention à l'expérience des personnes migrantes. En effet, plutôt que de collecter objets et témoignages, l'ouvrage s'appuie sur des sources de seconde main, rapports d'ONG et publications académiques. $\mathrm{Si}$ la bibliographie est très riche, les développements théoriques, souvent excessivement complexes, sont comme en suspension. 
C'est l'exposition présentée au musée Pitt-Rivers d'Oxford (27/04/2019-29/11/2019) qui donne un ancrage matériel indispensable à l'essai théorique. L'ouvrage et l'exposition gagnent en effet à être envisagés ensemble. L'exposition présente une série d'objets et d'œuvres d'art collectés auprès de personnes exilées, de bénévoles et d'artistes. Un long travail de coconstruction de l'exposition a été effectué, en particulier par Sarah Mallet, regroupant une dizaine de hauts-commissaires de l'exposition qui ont travaillé, au cours d'ateliers, au choix des œuvres et à leur présentation. La série photographique de Hank Wildschut montre ainsi l'évolution du campement, à travers les images d'un même emplacement sur lequel on voit d'abord des tentes et une cabane de bois, puis trois cabanes construites avec des matériaux standardisés (apportés par les organisations humanitaires), puis les marques des fondations de ces cabanes après leur destruction, enfin les traces des engins de terrassement qui ont effacé les derniers signes d'habitation. L'œuvre d'un artiste soudanais anonyme montre la violence policière avec la puissance d'un Basquiat. Des dessins d'enfants réalisés lors d'ateliers artistiques organisés par les bénévoles côtoient les œuvres d'artistes venus dans la «jungle » pour témoigner de ses réalités, comme Anne Gorouben, qui livre ses portraits délicats, ou Carine Bazin, qui documente à la fois la fragilité des architectures éphémères et leurs habitants (Traversant). Des dizaines de photographies constituent une archive inestimable de la "jungle ", complétée par des vidéos accessibles en ligne sur le site de l'exposition. L'artiste iranien Majid Adin - qui a vécu plusieurs mois dans la «jungle» avant de parvenir à traverser la frontière - a produit une œuvre spécifiquement pour l'exposition, superposant les silhouettes des migrants dans le quotidien du campement et une photographie du paysage hostile (The Hopeland). Un morceau de grillage, des grenades lacrymogènes rappellent dans l'exposition cet environnement matériel, où la force publique fait usage de violence contre le passage illégal. Le mobilier de l'école renvoie à la solidarité organisée par des citoyens et bénévoles venus en masse de Grande-Bretagne, mais aussi de France, de Belgique, d'Allemagne, d'Espagne et d'ailleurs, et qui se sont efforcés d'humaniser le lieu, d'en faire autre chose qu'un campement de transit pour clandestins, de le faire reconnaître comme un lieu de vie.

6 Pour répondre à la question "que reste-t-il du campement de la Lande ? », un travail participatif a donc été engagé. Cette démarche s'inspire de l'archéologie collaborative que Rachael Kiddey a pratiquée avec un groupe de personnes sans-abri et une équipe de recherche à Bristol, soit une "pratique matérielle et créative impliquant des allersretours entre la culture matérielle (paysage, lieux, objets) et l'héritage immatériel (souvenirs, récits, expériences) ${ }^{8} »$. Le travail muséographique entrepris transfigure la « jungle » en un «monument », un lieu de mémoire autour duquel se reconstruisent les identités blessées par la violence des frontières.

7 C'est ainsi que les auteurs expliquent leur démarche: « Comprendre le paysage non pas seulement comme un camp de réfugiés, mais comme une manifestation monumentale contre la frontière 9 . » Cette formule saisit parfaitement la particularité de la «jungle » de Calais de 2015-2016 en tant qu'événement et spectacle, produit d'une surpolitisation et d'une surmédiatisation de la crise des frontières en Europe, dont les conséquences ne cessent de se faire sentir sur les politiques migratoires et la croissance de mouvements xénophobes. Aucun campement n'aura donné lieu à autant d'images, au point que certains habitants du bidonville indiquaient par un pictogramme sur leur cabane qu'ils ne souhaitaient pas être photographiés. Dans le même temps, cette survisibilité aura 
été une opportunité pour les réfugiés d'intervenir dans l'espace public, d'y porter leur voix et leurs revendications, se saisissant de la "jungle" comme de la "sphère d'apparition (sphere of appearance) » d'une performativité collective ${ }^{10}$. Au moment du démantèlement, la préfecture, qui organisait cette opération "humanitaire " d'expulsion, de dispersion et de mise à l'abri, a distribué pas moins de sept cents accréditations de presse. Reportages, documentaires, films de fiction, romans, bandes dessinées, œuvres picturales, etc. ont contribué à produire pléthore d'images et de récits de ce que fut cette expérience éphémère. Plusieurs projets anthologiques ou muséographiques ${ }^{11}$ ont collecté ces éléments de culture matérielle et immatérielle: celui du Pitt-Rivers apporte une nouvelle pièce à l'édifice mémoriel, comme acte de résistance et d'endurance. Dans Après les camps, Jean-Frédéric de Hasque et Clara Lecadet soulignent cet enjeu nouveau d'une mémorialisation de l'expérience du déplacement et de l'encampement ${ }^{12}$. La patrimonialisation du campement, si elle peut relever d'une démarche historique, peut aussi être le support d'imaginaires plus ou moins fantasmés ${ }^{13}$. L'attention minutieuse aux objets de la migration et à leurs usages ${ }^{14}$ constitue alors un ancrage empirique et un garde-fou méthodologique contre des théorisations qui iraient trop « au-delà » de leur sujet.

\section{NOTES}

1. L'autrice de ces lignes y a contribué, à travers une bande dessinée (Lisa Mandel et Yasmine Bouagga, Les Nouvelles de la jungle, Bruxelles, Casterman, 2017), et deux ouvrages collectifs (Collectif Babels, De Lesbos à Calais : comment l'Europe fabrique des camps, Neuvy-en-Champagne, Le Passager clandestin, 2017 ; Michel Agier, Yasmine Bouagga et al., La Jungle de Calais, Paris, Puf, 2018).

2. À Calais, une équipe de l'École nationale supérieure d'architecture de Paris-Belleville dirigée par Cyrille Hanappe et Albert Hassan a procédé aux relevés des constructions de la «jungle» de Calais (2016). Le camp de Zaatari en Jordanie a fait l'objet d'études particulièrement riches sur l'habitat (voir Kamel Doraï et Pauline Piraud-Fournet, « From Tent to Makeshift Housing: A Case Study of a Syrian Refugee in Zaatari Camp (Jordan) », in Mona Fawaz et al., Refugees as City-Makers, Beyrouth, American University of Beirut, 2018 : 136-139).

3. Communication personnelle des auteurs, Oxford, 21 octobre 2019.

4. Jason De León, The Land of Open Graves: Living and Dying on the Migrant Trail, Oakland, University of California Press, 2015.

5. Voir l'enquête du collectif [en ligne], disponible sur: https://forensic-architecture.org/ investigation/the-left-to-die-boat (consulté le 08/06/2020).

6. Comme l'indiquent les auteurs (p. 84-85), «chaque exemple de collecte, de fabrication, de photographie, de publication sur Internet, de dessin, de peinture, de conservation, est, dans une certaine mesure, un exercice visant à redessiner la frontière britannique - chaque fois plus durable dans la mesure où il est plus humain que les clôtures, les passeports, la sécurité frontalière, les centres de rétention administrative et le reste de l'infrastructure produisant la frontière. Une telle opération de contre-cartographie adopte une forme non géométrique. Elle est une cartographie interventionniste pour une cosmopolitique de la mémoire.» (Traduit par l'autrice.) 
7. Archives de la Ville de Calais, H96(26M).

8. Rachael Kiddey, Homeless Heritage: Collaborative Social Archaeology as Therapeutic Practice, Oxford, Oxford University Press, 2017 : I. (Traduit par l'autrice.)

9. ibid. : 77. (Traduit par l'autrice.)

10. Judith Butler, Notes Toward a Performative Theory of Assembly, Cambridge (États-Unis), Harvard University Press, 2016 : 58, citée dans l'ouvrage. (Traduit par l'autrice.)

11. Par exemple : "Call Me By My Name: Stories from Calais and Beyond», Londres, Migration Museum, 2017 ; «Évasions », Sète, MIAM, 2018 ; «Réinventer Calais », Pontault-Combault, CPIF, 2019 ; «Calais. Témoigner de la "jungle” », Paris, Centre Georges-Pompidou, 2019.

12. Jean-Frédéric de Hasque et Clara Lecadet (dir.), Après les camps : traces, mémoires et mutations des camps de réfugiés, Louvain-La-Neuve, Academia/L'Harmattan, 2019.

13. Alexandra Galitzine-Loumpet, "Le livre de "la jungle de Calais": imaginaires et désubjectivations", Journal des anthropologues, $\mathrm{n}^{\circ}$ hors-série ("Subjectivités face à l'exil: positions, réflexivités et imaginaires des acteurs »), $2018:$ 95-123.

14. Voir le programme de recherche Migrobjets [en ligne], disponible sur: https:// migrobjets.hypotheses.org/ (consulté le 08/06/2020).

\section{AUTEURS}

\section{YASMINE BOUAGGA}

Yasmine.bouagga[at]ens-lyon.fr 